\title{
IMPLEMENTATION OF PREOXIDATION IN CONVENTIONAL POTENTIATING PLANT PILOT SCALE FOR THE REDUCTION OF DISSOLVED ORGANIC MATTER IN SURFACE WATERS
}

\author{
IMPLEMENTACION DE LA PREOXIDACION EN PLANTA \\ POTABILIZADORA CONVENCIONAL ESCALA PILOTO PARA \\ LA REDUCCION DE MATERIA ORGANICA DISUELTA EN \\ AGUAS SUPERFICIALES
}

\author{
Vera. M, D. E. ${ }^{*}$ Viracachá. Q, L. $F_{4}^{2}$ Lopez. A, L.A. ${ }^{3}$ Ramón. V, J. A. ${ }^{4}$ \\ 1 Ing. Derly Estefany Vera Mogollón, Ingeniera Ambiental. \\ Profesional Operativo Proyecto SATC e-mail: veraderly05@gmail.com \\ ${ }^{2}$ MsC. Luis Fernel Viracachá Quintero. Ingeniero Civil, Universidad \\ Industrial de Santander, e-mail: lufernel@hotmail.com \\ ${ }^{3}$ Ing. Lizeth Amparo López Areniz. Ingeniera Química, Universidad \\ Industrial de Santander. Jefe de Planta de Tratamiento \\ EMPOPAMPLONA S.A.E.S.P, e-mail: \\ ing.plantas@empopamplonasa.gov.co \\ ${ }^{4}$ Ph. D. Jacipt Alexander Ramón V. Director Proyecto SATC, e-mail: \\ jacipt@unipamplona.edu.co
}

\begin{abstract}
Water destined for consumption is subjected to several purification processes depending on the quality of the source, among the processes characteristic of a plant can include coagulationflocculation, sedimentation, filtration, disinfection; In the present investigation it is intended to include the pre-oxidation process as a pretreatment with the objective of facilitating the reduction of total organic carbon (TOC) and dissolved organic carbon (DOC) present in the water supply, taking into account that these are parameters used to determine The amount of organic substances in surface waters. In order to carry out the research it is necessary to carry out a preliminary evaluation of the behavior and the physicochemical properties of the water, in addition to the variation of the turbidity through the humic and fulvic acid device taking into account the ranges for each of the levels of complexity Established by RAS 2015 and subsequent determination of the optimal dose of preoxidants (potassium permanganate and hydrogen oxide) and coagulants (PAC, aluminum sulfate and ferric chloride). Through the implementation of preoxidation it is expected to obtain a remarkable efficiency in the reduction of the organic matter present in the resource due to the reactions that are carried out and the characteristic properties of the preoxidants used, besides the contribution of these to the coagulation Of suspended matter and colloids; It is also intended to demonstrate that it is possible to potabilize water with the inclusion of the pre-oxidation process in an efficient and efficient way, obtaining the liquid with the conditions suitable for human consumption without generating adverse effects on human health.
\end{abstract}


Key words: surface water, dissolved organic carbon, potabilization, preoxidant,

\section{RESUMEN}

El agua destinada para consumo se somete a diversos procesos de potabilización dependiendo de la calidad de la fuente, entre los procesos característicos de una planta potabilizadora se pueden incluir coagulación-floculación, sedimentación, filtración, desinfección; en la presente investigación se pretende incluir el proceso de preoxidacion como pretratamiento con el objetivo de facilitar la disminución de carbono orgánico total (COT) y carbono orgánico disuelto (COD) presente en el agua de abastecimiento teniendo en cuenta que estos son parámetros utilizados para determinar la cantidad de sustancias orgánicas presentes en las aguas superficiales. Para llevar a cabo la investigacion es necesario realizar una evaluación previa del comportamiento y propiedades fisicoquímicas del agua, además de la variación de la turbidez mediante la aplicación de ácidos húmicos y fulvicos teniendo en cuenta los rangos para cada uno de los niveles de complejidad establecidos por el RAS 2015 y la posterior determinación de dosis optima de preoxidantes (permanganato de potasio y peróxido de hidrogeno) y coagulantes (PAC, sulfato de aluminio y cloruro férrico). Mediante la implementación de la preoxidación se espera obtener una eficiencia notable en la reducción de la materia orgánica presente en el recurso debido a las reacciones que se llevan a cabo y las propiedades características de los preoxidantes utilizados, además de la contribución de estos a la coagulación de materias en suspensión y coloides; además se pretende demostrar que es posible potabilizar el agua con la inclusión del proceso de preoxidacion de una manera eficiente y eficaz obteniendo el líquido con las condiciones aptas para el consumo humano sin generar efectos adversos en la salud humana.

Palabras clave: agua superficial, carbono orgánico disuelto, potabilización, preoxidante, turbidez.

\section{INTRODUCCIÓN}

Las actividades que diariamente realiza el hombre produce ciertas cantidades de contaminantes incorporándose al recurso hídrico y generando alteraciones en él y sus propiedades, provocando contaminación en ella que la hacen no apta para el consumo humano; actualmente existen diferentes técnicas, procesos y sustancias que permiten potabilizar el agua con el propósito de tratar el agua y eliminar características indeseables, impurezas, sustancias toxicas y agentes patógenos a fin de proporcionar agua segura, agradable y aceptable a los consumidores dando cumplimiento a las normas de calidad establecidas
Las normas establecidas para dar cumplimiento a parámetros de calidad y criterios de sanidad cada vez son más estrictas, por tal razón la selección de uno o más procesos que se deben incluir en la potabilización es una gran responsabilidad resultado de una tarea compleja debido al cumplimiento que se debe darna las exigencias de dichas normas. La elección correcta de un proceso de tratamiento del agua está determinada por la calidad inicial del recurso hídrico, por la calidad que se desea obtener que está relacionada con los rangos dé cada uno de los parámetros reglamentados por la ley y el cumplimiento de objetivos de calidad, además de la satisfacción de necesidades y aporte al mejoramiento de calidad de vida de los consumidores. (Rojas 2008; Vanegas et al, 2017). 
Con el objetivo de obtener agua potabilizada (con las condiciones óptimas para el consumo humano) a partir de una fuente natural es necesario tener en consideración las características físicas, químicas y microbiológicas debido a su influencia en cuanto a aceptación de acuerdo a su calidad (Mejía, 2010; Angulo et al, 2017). Para cumplir con los rangos establecidos por el RAS al agua superficial captada se le deben realizar una cantidad de procesos característicos de las plantas potabilizadoras convencionales como lo son coagulación, floculación, sedimentación, filtración y desinfección, adicionando un proceso de pre oxidación posterior a la captación con el objetivo de reducir la materia orgánica presente en el recurso hídrico. Los procesos nombrados anteriormente y demás que se incluyan en el tratamiento para la potabilización de recurso están encaminados a retirar todos los elementos, sustancias y demás que puedan llegar generar malestar en el ser humano.

La inclusión del proceso de pre oxidación en el tratamiento para la potabilización del agua se justifica en cuanto se refiere a que este proceso ayuda a la disminución de la cantidad de materia orgánica presente en el agua, permitiendo así que las cantidades de carbono orgánico disuelto que lleguen a los siguientes procesos de plantas potabilizadoras convencionales (coagulación, floculación, sedimentación, filtración y desinfección) sean mucho menores, obteniendo mayor eficiencia en el tratamiento y menores posibilidades de formación de subproductos de desinfección.

\section{MARCO TEÓRICO}

Una planta de tratamiento de agua potable es el conjunto de instalaciones, destinadas a mejorar la calidad del agua. Frecuentemente, la totalidad de los tratamientos que se efectúan sobre el agua de un abastecimiento se realizan en la planta de tratamiento. Sin embargo, en ocasiones, se realizan otros tratamientos fuera de la planta, ya sea en la captación (desbaste grueso o fino, desarenado, predecantación, recloración), e incluso en la red de distribución. (Casero R, 2007; Espinel et al, 2016).

El agua destinada para consumo puede ser sometida a diversos tratamientos, que pueden incluir procesos de plantas potabilizadoras convencionales tales como captación, coagulación-floculación, sedimentación, filtración, desinfección entre otros, la inclusión de estos está determinada por las características iniciales de la fuente y la calidad del agua.

Para dar cumplimiento a las normas de calidad de agua se han establecido diferentes tratamientos con el fin de potabilizar el recurso, siendo las plantas potabilizadoras convencionales las más comunes

Entre los procesos de las plantas potabilizadoras convencionales se encuentran los siguientes

\section{Coagulación}

Junto con la floculación son Procesos fisicoquímicos para mejorar la eficiencia de reducción de material particulado y de coloides de los siguientes procesos de sedimentación o filtración. La coagulación incluye la dosificación de sustancias químicas para desestabilizar las partículas suspendidas con cargas similares. Esto permite que se unan y que se inicie la formación de flóculos. (Perez Parra, 2009; Rodríguez, 2017).

\section{Floculación}

Proceso que comprende una agitación lenta del agua por un período relativamente largo, durante el cual las partículas finamente divididas o en estado coloidal, van juntándose - aglomerándose para formar un flóculo hidratado de tamaño tal que puedan 
sedimentar bajo la acción gravitatoria. Sin embargo, los flóculos más finos no son eliminados por sedimentación y por lo tanto hay que recurrir a la filtración como proceso complementarios posteriores a la coagulación. [5]. (Orellana, 2005; Gutiérrez et al, 2016).

\section{Sedimentación}

La sedimentación es la remoción de sólidos en suspensión en un fluido por la fuerza de gravedad. Los sólidos pueden estar presentes en el agua en la forma como ocurren naturalmente, como es el caso del limo o la arena, o en forma modificada de su estado natural, como resultado de la coagulación y la floculación. Si las partículas son más densas que el agua mayor es su probabilidad de sedimentación, dando como resultado un fluido clarificado, y en el fondo de los tanques una suspensión más concentrada que se considera ha sido separada del mismo. (Perez Parra, 2009; Velandia et al, 2016) La sedimentación después de la adición de coagulante y de la floculación se usa para remover los sólidos sedimentables que han sido producidos por el tratamiento químico como en el caso de remoción de color y turbiedad. (Romero, 2000; Melo et al, 2017).

\section{Filtración}

La filtración consiste en la remoción de partículas suspendidas y coloidales presentes en una suspensión acuosa que escurre a través de un medio poroso. En general, la filtración es la operación final de clarificación que se realiza en una planta de tratamiento de agua y, por consiguiente, es la responsable principal de la producción de agua de calidad coincidente con los estándares de potabilidad. (Maldonado Yactayo, 2009; Villamizar y Justinico, 2017)

\section{MATERIALES Y MÉTODOS}

\subsection{Diseño de la planta}

Para el desarrollo de la investigación se realizó el diseño de una planta potabilizadora convencional teniendo en cuenta lo estipulado en el RAS 2015 título C para dosis (coagulante y desinfectante), pruebas de tratabilidad, diseño de las unidades que compone la planta ( resalto hidráulico para mezcla rapida, floculador, sedimentador, filtro, resalto para desinfección), además se tuvo en cuenta la información referente a preoxidacion, ya que es el proceso elegido para reducción de subproductos de desinfección.

\subsection{Analizar la influencia de la calidad del afluente a tratar, en la eficiencia del proceso de potabilización por medio de un tratamiento convencional}

Para cumplir los objetivos planteados inicialmente se realizó un análisis detallado del comportamiento del agua supetrficial a utilizar como fuente, evaluación que se realiza mediante la medición de parámetros de calidad establecidos utilizando métodos estandarizados como : turbidez, solidos, acidez, alcalinidad, dureza, $\mathrm{pH}$, conductividad eléctrica, cloruros, oxígeno disuelto, color, sulfatos, DQO, DBO, nitratos, fosfatos, entre otros. (APHA, 1995).

Los análisis se realizaron en las instalaciones del laboratorio de control y calidad de la Universidad de Pamplona y el laboratorio de aguas de la empresa EMPOPAMPLONA en la ciudad de Pamplona.

\subsection{Estimar las dosis optimas de pre oxidante para diferentes concentraciones de materia orgánica que permita la reducción de subproductos de la desinfección con cloro.}

Para implementar la preoxidación como pretratamiento se hizo necesaria la utilización de agentes oxidantes, para ello se usaron 2 compuestos con propiedades oxidantes como lo son el permanganato de potasio y 
peróxido de hidrogeno, su uso se debe cumplir con lo establecido el RAS 2015 y La NTC 2753.

De acuerdo a los ensayos realizados para determinación del preoxidante óptimo se estimaron eficiencias mayores para la preoxidación con permanganato de potasio, por lo cual posteriores análisis se realizaran con la utilización de dicho oxidante, teniendo en cuenta lo recomendado en el Titulo $\mathrm{C}$ del RAS para la Oxidación por $\mathrm{KMnO} 4$ :

Los desinfectantes al igual los oxidantes y coagulantes se aplican mediante bombas dosificadoras peristálticas. Terminado el proceso de potabilización se procede a medir los parámetros fisicoquímicos y verificar la calidad del agua, además de realizar mediciones de carbono orgánico disuelto y determinar la eficiencia que ha tenido la preoxidacion

\subsection{Establecer criterios de diseño para reducción de materia orgánica disuelta en una planta potabilizadora convencional utilizando permanganato de potasio y peróxido de hidrogeno}

De acuerdo a la literatura la DQO está relacionada con la cantidad de carbono presente en el recurso hídrico, por lo cual se hacen mediciones de este parámetro para obtener un análisis cuantitativo de la variación del COT presente en el recurso, dependiendo del nivel de calidad, después de realizar el proceso de preoxidación y finalmente al agua potabilizada, adicionalmente se realizaron análisis termogravimétricos (análisis cualitativos ) ya que esta es una técnica de análisis térmico que estudia el cambio de masa de una muestra en función del tiempo a temperatura constante o en función de la temperatura. Dicho cambio puede ser debido a reacciones químicas, procesos de descomposición.
Otro análisis cualitativo implementado es la espectroscopia de infrarrojo, este tipo de espectroscopia se basa en la absorción de la radiación infrarroja por las moléculas en vibración. En una molécula, todos los átomos vibran alrededor de la distancia interatómica media. Existen dos modos principales de vibración, alargamiento y flexión, y éstos están cuantizados Una molécula absorberá energía cuando ésta sea igual a la necesaria para que se produzca una transición vibracional de la molécula, dichos análisis se realizan en el laboratorio de biocalorimetria de la Universidad de Pamplona..

\section{RESULTADOS Y DISCUSIÓN}

\subsection{DISEÑO DE LA PLANTA}

El caudal de agua cruda (QAC) para el diseño de la planta de tratamiento de agua potable se determinó teniendo en cuenta el cumplimiento que se debe dar a lo establecido por el RAS, además se tiene en cuenta que la bomba utilizada para impulsar el líquido no trabaje a su máxima capacidad, además de no generar un diseño de una PTAP demasiado grande lo cual aumentaría en costos para su construcción, funcionamiento y mantenimiento, por lo tanto el caudal de diseño seleccionado, de este modo será: $\mathrm{QAC}=0.2 \mathrm{l} / \mathrm{s}$.

La bomba que suministrara el agua cruda a la planta de tratamiento de agua potable se puede observar en la imagen 1

Imagen 1. Bomba impulsora 


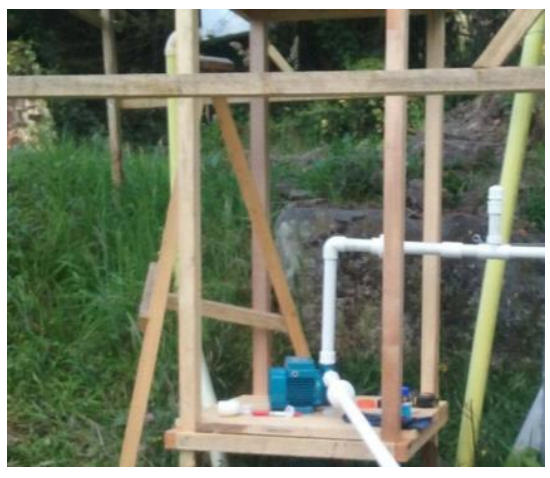

Fuente. Autores 2017

A la planta escala laboratorio previamente diseñada (2013) utilizada como base para el desarrollo de la presente investigación se le adiciona una unidad: tanque de preoxidación,

\subsubsection{TANQUE DE PREOXIDACION}

Imagen 2. Tanque de preoxidación

Fuente. Autores 2017

Para la elección del tanque de preoxidación se debe tener en cuenta el tiempo de retención, que está dado por el tiempo en que se da la oxidación de la materia orgánica de acuerdo a ensayos se evidencio que la preoxidación es más eficiente en pHs neutros a ligeramente ácidos por lo cual se consideran $\mathrm{pHs}$ mayores a 6 y cercanos a pHs neutros, teniendo en cuenta que la fuente generalmente es de carácter neutro a básico, se hizo necesaria la aplicación de $\mathrm{H} 2 \mathrm{SO} 4$, acidificando la muestra.

De acuerdo al RAS el tiempo de reacción para el agua de carácter neutro a ligeramente acido, es de 1,5 a $4 \mathrm{~min}$, teniendo en cuenta esto se realizan ensayos a un $\mathrm{pH}$ de 6,5 obteniendo como resultado que las eficiencias obtenidas para un tiempo de reacción de 3 min y 4 min no difieren, por lo cual se toma como tiempo de retención 3 minutos

\subsubsection{DESCRIPCIÓN DE LA PLANTA}

En la imagen 3 se observa la captación que se hace por parte de EMPOPAMPONA S.A.E.S.P , posteriormente el recurso se conduce hacia el Desarenador, la fuente utilizada para potabilizar en el caso de estudio es desarenada, debido a que no hay forma de conducirlo por gravedad y a que los niveles de la lámina de agua varían, se realiza una captación para la planta escala laboratorio con una bomba impulsora, posteriormente se implementa la unidad que permite realizar la preoxidación y seguidamente la coagulación, estos dos procesos (dosificación de oxidantes y coagulantes se realiza con bombas dosificadoras, del floculador el agua se conduce hacia el sedimentador y luego hacia filtro de flujo descendente. Donde finalmente se realiza la desinfección y evaluación de calidad de agua. 
REVISTA AMBIENTAL

a

AGUA, AIRE Y SUELO 

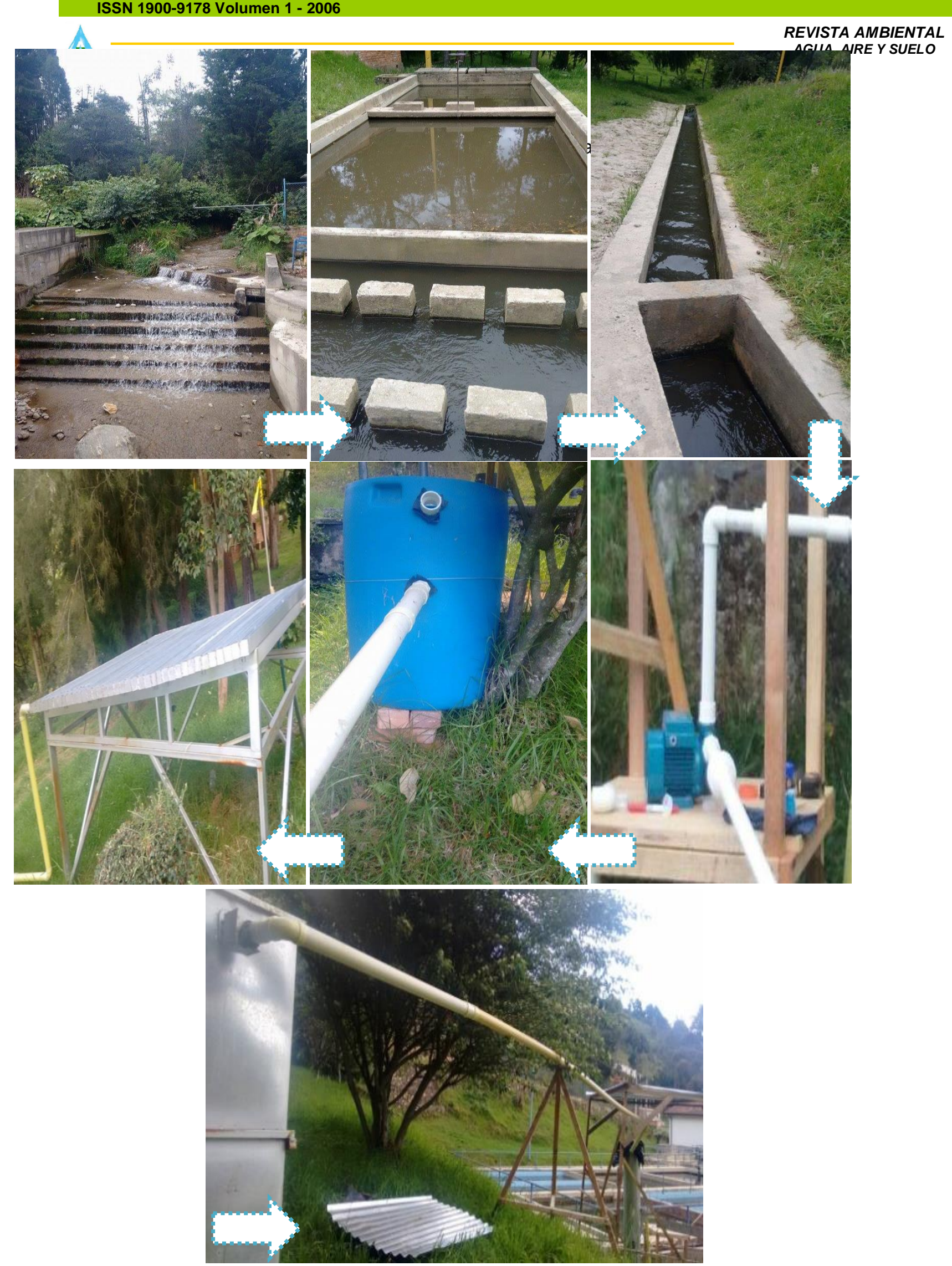

Fuente. Autores 2017 


\subsection{EVALUACION DEL COMPORTAMIENTO DEL AGUA}

Para la evaluación del comportamiento del agua natural se realizaron ensayos de caracterización a la fuente a las muestras tomadas teniendo en cuenta los siguientes parámetros

\subsubsection{Turbidez}

La medición de la turbiedad se realiza mediante un turbidímetro o Nefelómetro Las unidades utilizadas son, por lo general, unidades nefelométricas de turbiedad (UNT).

En cuanto a la turbidez de la fuente se evidencia que la fuente puede calificarse como regular, teniendo en cuenta los rangos establecidos por el RAS, de acuerdo a la Figura 1, los valores de turbidez son mayores a 2 y menores a 40 .

Figura 1. Turbidez del agua natural

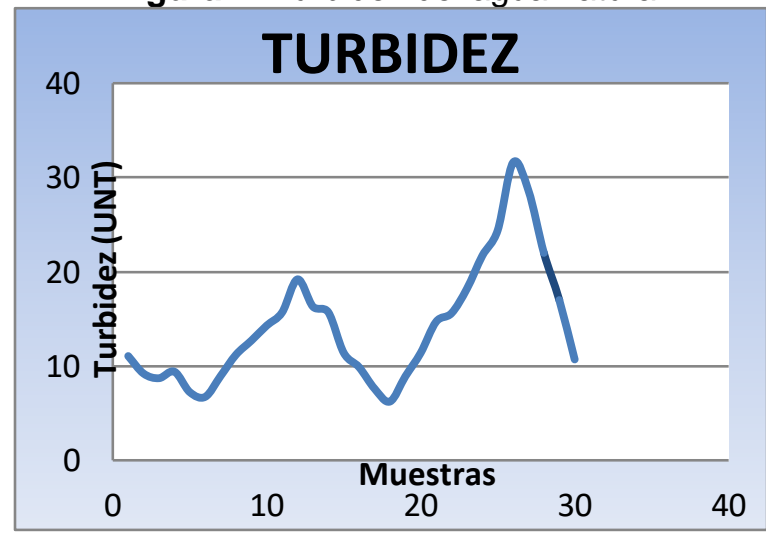

Fuente. Autores, 2017.

\subsubsection{Dureza}

Debido a las sales de magnesio y calcio en la fuente utilizada puede considerarse como muy dura, (Figura 2), dichas concentraciones dependen fundamentalmente de las formaciones geológicas atravesadas por el agua de forma previa a su captación.
Figura 2. Dureza en el agua

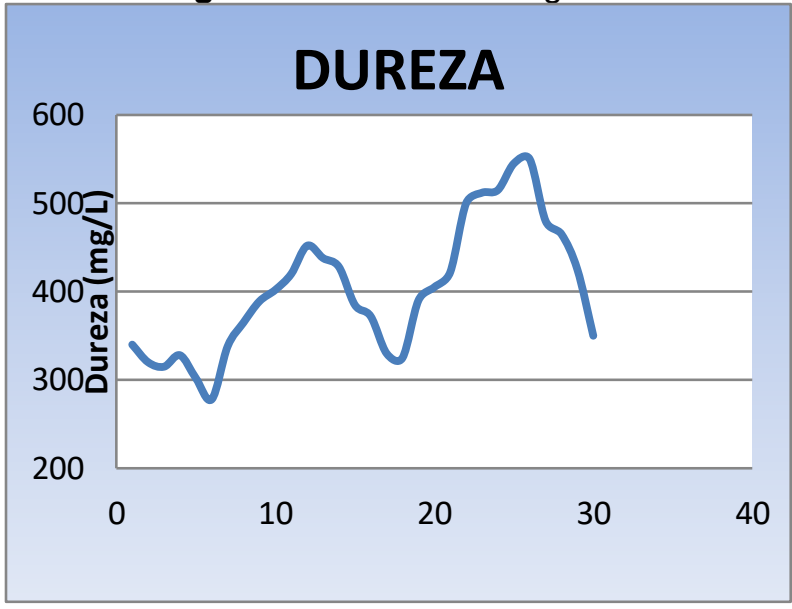

Fuente. Autores, 2017.

\subsubsection{Oxigeno disuelto}

Es el más importante agente oxidante del agua natural. El oxígeno disuelto es importante en los procesos de: fotosíntesis, oxidación-reducción, solubilidad de minerales y la descomposición de materia orgánica.

En un cuerpo de agua se produce y a la vez se consume oxígeno. La producción de oxígeno está relacionada con la fotosíntesis, mientras el consumo dependerá de la respiración, descomposición de sustancias orgánicas y otras reacciones químicas.

Figura 3. Oxígeno disuelto del agua natural

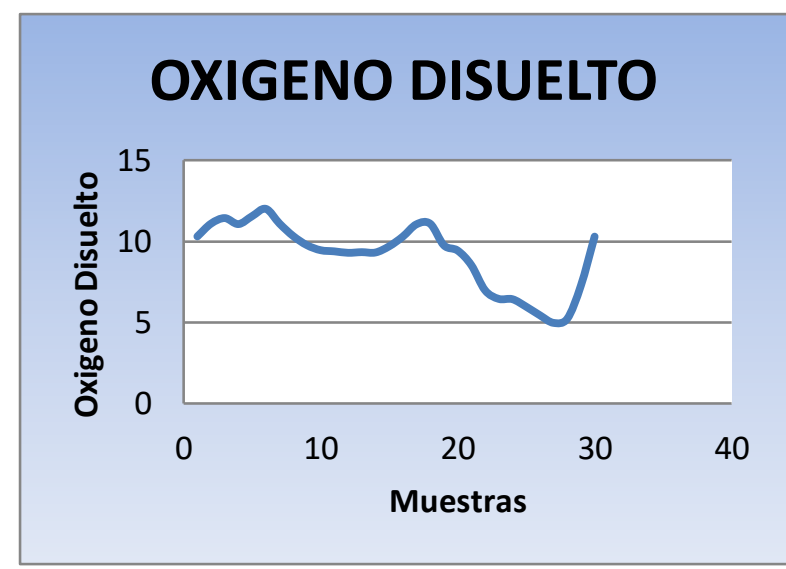

Fuente. Autores, 2017.

\subsection{4. $\mathrm{pH}$}

Las muestras analizadas evidencian que el recurso utilizado es de carácter neutrobásico, debido al contenido de carbonatos, bicarbonatos e hidróxidos, algunas sales de 
ácidos débiles. Teniendo en cuenta la tabla C.2.1. De calidad de la fuente del RAS, teniendo e cuenta los rangos establecidos de $\mathrm{pH}$ promedio para los 4 niveles de complejidad, la fuente puede ser caracterizada como aceptable, regular 0 deficiente. $(\mathrm{pH}=7,5046667)$

Figura 4. $\mathrm{pH}$ del agua natural

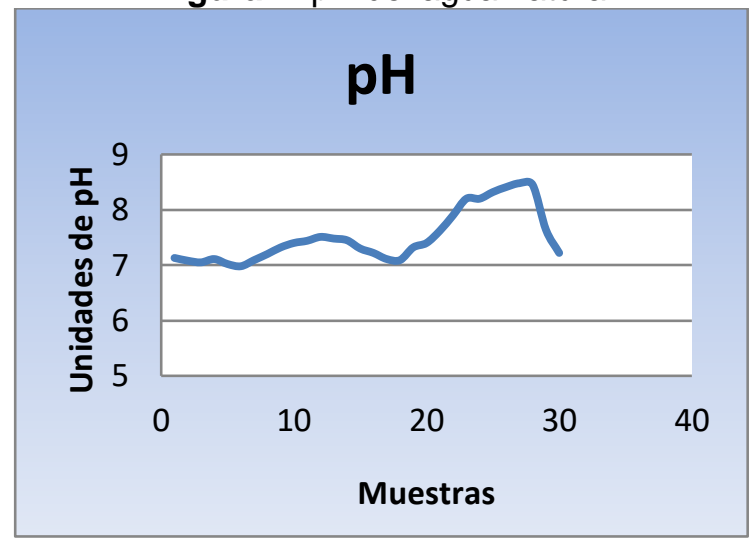

\subsubsection{Conductividad eléctrica}

De acuerdo a las Figuras 5 y 6 se estima que los cloruros son los principales contribuyentes a la conductividad eléctrica que presenta la fuente.

Figura 5. Conductividad eléctrica del agua natural

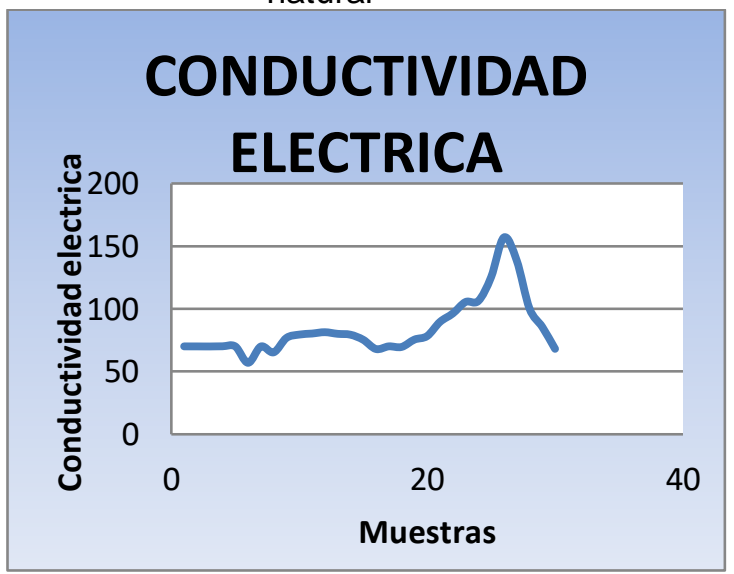

Fuente. Autores, 2017.
Figura 6. Cloruros del agua natural

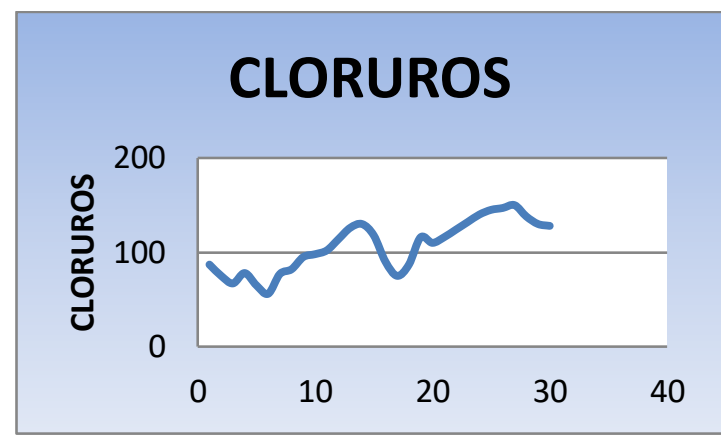

Fuente. Autores, 2017.

\subsubsection{Color}

Usualmente el color se asocia a la calidad del agua, ya que colores inusuales en el recurso se relacionan con contaminación por productos químicos, sustancias orgánicas, puede indicar la presencia de bacterias y algas.

Figura 7. Color del agua

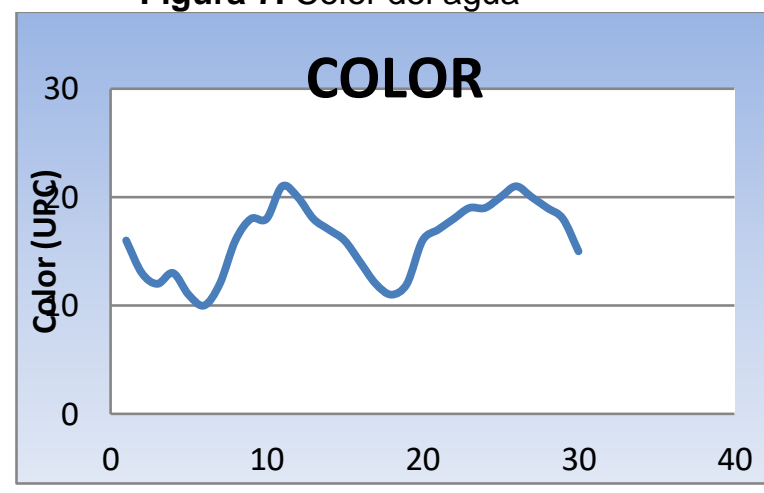

Fuente. Autores, 2017.

Los rangos de UPC obtenidas en los ensayos de color para la fuente de abastecimiento indican que el agua puede ser catalogada como fuente regular, debido a que los valores reflejados se encuentran entre 10-20 UPC

\subsubsection{Cloruros}




\subsubsection{Temperatura}

Figura 8. Temperatura del agua natural

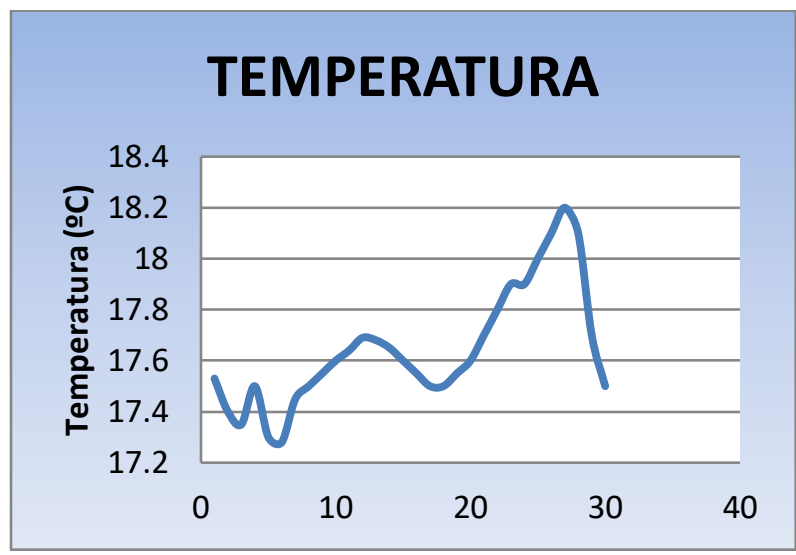

Fuente. Autores, 2017.

La temperatura está relacionada con la conductividad ya que esta última depende en parte de la temperatura a la que se encuentra el agua. A mayor temperatura, mejor conductividad. Además al provocar resistencia a las corrientes eléctricas, esta energía aumentará la temperatura del agua. A su vez, la temperatura está muy relacionada a la turbidez, ya que si la turbidez es alta, la radiación solar es absorbida por las partículas que hay en el agua y esta la calienta

\subsection{DETERMINACION DE DOSIS DE ACIDOS HUMICOS Y FULVICOS}

Teniendo en cuenta la metodología de la investigación en desarrollo, basada en establecer criterios de diseño para reducción de materia orgánica disuelta en una planta potabilizadora convencional teniendo en cuenta los diferentes niveles de compleidad establecidos en el RAS, se propone alterar la turbidez de la fuente mediante la aplicación de sustancias húmicas y fúlvicos, materiales orgánicos heterogéneos.
Según la tabla 1, la fuente es catalogada como Fuente Regular, de acuerdo a estos resultados se tiene que no se puede obtener la fuente aceptable, por lo tanto, se realizaron ensayos aplicando dichas sustancias al $1 \%$ con diferentes dosis.

De acuerdo a ensayos de laboratorio realizados para establecer dosis de laboratorio que permita obtener el recurso en los diferentes niveles de complejidad se obtuvieron 4 muestras representativas con las cuales se determinó que las respectivas dosis de ácidos húmicos y fúlvicos al $5 \%$ serán las siguientes

Tabla 1. Dosis de ácidos húmicos y fúlvicos

\begin{tabular}{|c|c|}
\hline $\begin{array}{c}\text { NIVEL DE } \\
\text { COMPLEJIDAD }\end{array}$ & $\begin{array}{c}\text { DOSIS DE ACIDOS } \\
\text { HUMICOS Y FULVICOS }\end{array}$ \\
\hline Regular & $\begin{array}{c}\text { Agua Naturales } \\
\text { Dosis } \leq 10 \mathrm{ml} / \mathrm{L}\end{array}$ \\
\hline Deficiente & Dosis $\geq 15 \mathrm{ml} / \mathrm{L} 0 \leq 100 \mathrm{ml}$ \\
\hline luy deficiente & Dosis $\geq 130 \mathrm{ml}$ \\
\hline
\end{tabular}

Fuente. Autores, 2017.

\subsection{PREOXIDANTE OPTIMO}

De acuerdo a la Figura 9, que representa el carbono orgánico total después de añadir los agentes oxidantes, se evidencia que las concentraciones removidas por el peróxido de hidrogeno son menores, por lo cual se elige el permanganato de potasio como preoxidante optimo, por lo cual es necesario realizar análisis de $\mathrm{pH}$, dosis $\mathrm{y}$ la concentración óptima para este compuesto, teniendo en cuenta lo establecido por las norma. 
Figura 9. COT KMnO4 VS H2O2

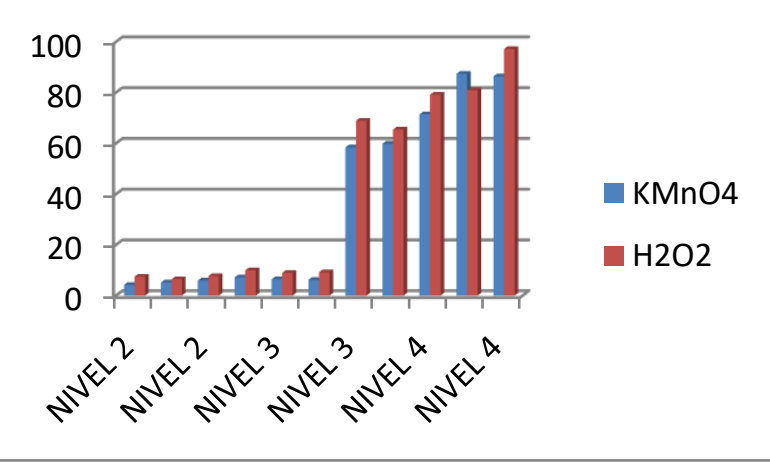

Fuente. Autores, 2017.

\subsection{ESPECTROSCOPÍA DE INFRARROJO POR TRANSFORMADA DE FOURIER}

Para la realización del análisis cualitativo de cantidades de carbono presentes en las diferentes muestras. Se llevaron a cabo estudios teniendo en cuenta espectroscopia IR, obteniendo los espectros para las muestras; a continuación se presentan la figura FT-IR para el nivel muy deficiente;

En la molécula de agua $(\mathrm{H} 2 \mathrm{O})$, el grupo funcional $\mathrm{O}-\mathrm{H}$ es el que contribuye a la frecuencia de resonancia (vibración) alrededor de los $3450 \mathrm{~cm}-1$.

Los datos espectrales de FT-IR Figura 10. de las muestras de agua indican la presencia de vínculos importantes; grupo éster, carbonilo, amino, hidroxilo, grupos funcionales muy inestables y otros picos característicos. Los picos mostrados en la banda de 1500 y 2350, no se caracterizaron como compuestos carbonados debido a que no se encuentra información al respecto en la literatura, de acuerdo a ciertos autores puede ser catalogado como ruido
Figura 10. Análisis FT-IR para muestra de nivel muy deficiente con $\mathrm{KMnO} 4$

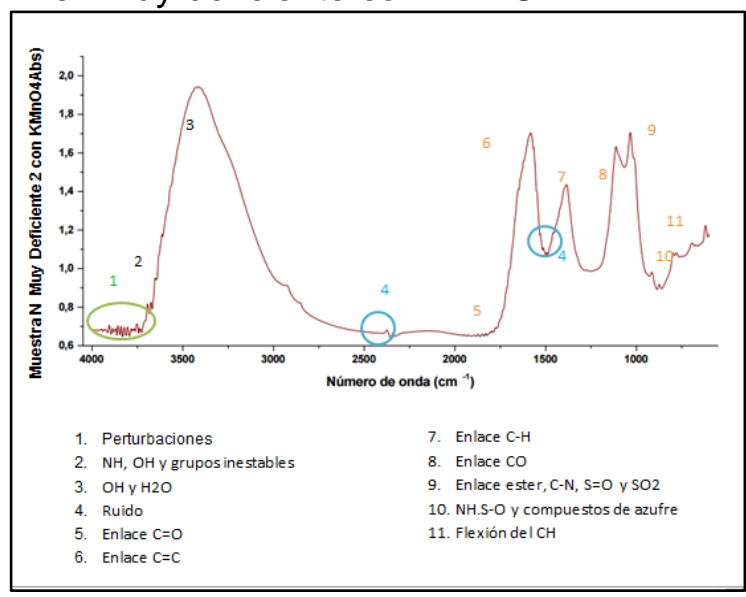

Fuente. Autores, 2017.

Una banda ancha en 3550-3200 cm-1 indica la presencia de grupos $\mathrm{OH}$, el grupo $\mathrm{OH}$ secundario de las moléculas en la cadena carbonada, (Cardeño, 2013)

Los picos en alrededor de 1800-1900 cm-1 representan la vibración de estiramiento para el grupo carbonilo $(C=O)$.

Una fuerte absorción debida a la vibración para $\mathrm{C}=\mathrm{C}$ puede ser asegurada por el pico a alrededor de $1625 \mathrm{~cm}-1$, mientras que en $1380 \mathrm{~cm}-1$ aproximadamente, se anticipa la vibración extendida por - $\mathrm{CH}$ (Patel et al, 2008)

La banda a $1380 \mathrm{~cm}^{-1}$ representa la deformación simétrica del $\mathrm{C}-\mathrm{H}$.

El pico que se observa alrededor de 1130 y 1200 evidencia la presencia de enlaces CO

También se puede identificar en la banda $1050 \mathrm{~cm}^{-1}$ compuestos con azufre $\mathrm{S}=\mathrm{O}$, SO2, además de enlaces éster y $\mathrm{CN}$. (Pouchert 1981).

Para bandas ubicadas en el rango 500$1000 \mathrm{~cm}^{-1}$ se puede encontrar enlaces $\mathrm{NH}$, S-O y otros compuestos de azufre. (Zaragodni et al. 2001).

En bandas inferiores menores a $500 \mathrm{~cm}^{-1} \mathrm{se}$ identificó la flexión del $\mathrm{CH}$. 


\subsection{ANALISIS \\ TERMOGRAVIMETRICO}

EI análisis termogravimétrico consiste en registrar continuamente la variación de la masa del material en estudio conforme se varía la temperatura a una tasa térmica constante (cambio de temperatura lineal). Como resultado del análisis termogravimétrico se obtienen los datos de cambio de masa con respecto a la temperatura o al tiempo y un termograma, el cual representa gráficamente las variaciones porcentuales de la masa. (Rodriguez, et al 2012)

Para la Figura 11 materia orgánica oxidada con permanganato de potasio y peróxido de hidrogeno la primera pérdida de peso se inicia en rangos de temperatura cercanos a los $100^{\circ} \mathrm{C}$ y evidencia la eliminación de agua que corresponde a un $12 \%$ de la muestra inicial. En temperaturas mayores hay pérdida de peso de aproximadamente $28 \%$ y se atribuye a la degradación de grupos sulfónicos, aminos, carbonilos, esteres, teniendo como referente lo analizado en las gráficas de espectrofotometría infrarroja

Figura 11. TERMOGRAVIMETRIA PARA MUESTRA MUY DEFICIENTE OXIDADA

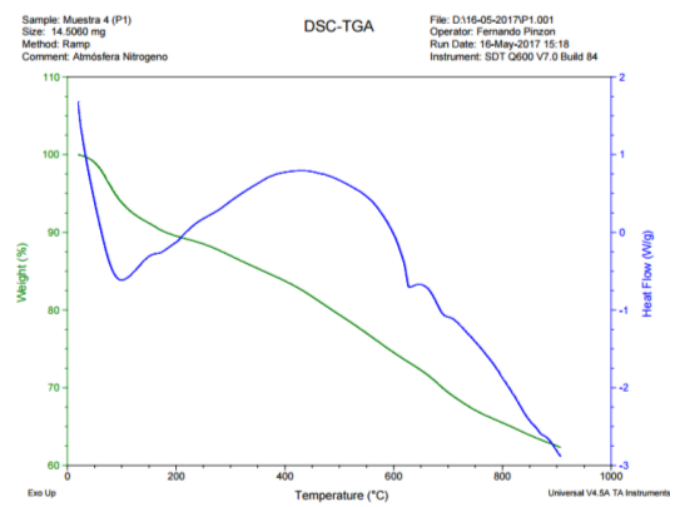

Fuente. Autores, 2017.

\subsection{DETERMINACION DE EFICIENCIA DE REMOCION DE CARBONO ORGANICO}

Figura 12. Variación del COT con utilización de oxidantes en diferentes niveles de complejidad

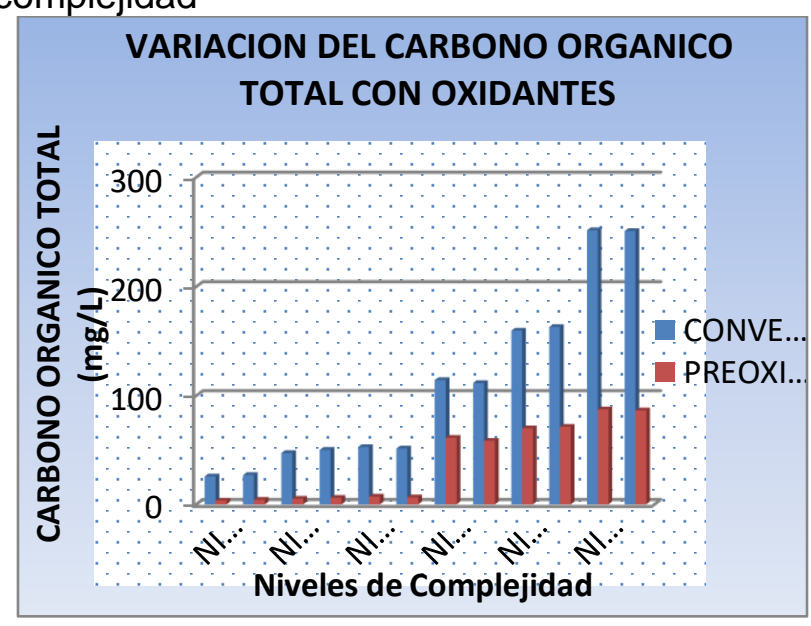

Fuente. Autores, 2017.

De acuerdo a la Figura 12, donde se muestra la variación del carbono orgánico total para el proceso convencional y la adición de la preoxidación con permanganato de potasio , se evidencia que la concentración del carbono orgánico para el recurso preoxidado disminuye notablemente con la adición de oxidantes, teniendo en cuanta las características oxidantes del $\mathrm{KMnO} 4$, con la adición de este se presenta mayor eficiencia en cuanto a la coagulación, y sedimentación debido a que el producto resultante en la reacción de oxidación del permanganato, el dióxido de manganeso, como sustancia insoluble, forma coágulos que favorecen la coprecipitación de materias en suspensión, coloides en el agua. También se comportan estos coágulos o flóculos como adsorbentes en el interior de los filtros de arena.

En aguas con alto contenido en materia orgánica, como la caracterizada para los niveles 3 y 4 de complejidad se ha comprobado la mejora en la coagulación y por tanto en la eliminación, tanto de turbidez, como de D.Q.O y por ende de COT Y COD. 


\section{CONCLUSIONES}

El diseño de una planta de potabilización de agua de funcionamiento hidráulico para tratar un caudal demasiado pequeño $(0,2 \mathrm{k} / \mathrm{s})$ es un proceso muy tedioso y arduo debido a que se deben cumplir recomendaciones del RAS, y el manejar un flujo de agua tan bajo se hace demasiado complicado llegar a cumplir con estas recomendaciones.

Se realizó la evaluación del comportamiento del agua superficial utilizada como fuente a potabilizar, adicionando un proceso como lo es el de pre oxidación de la materia orgánica el cual resulta eficiente ya que se obtuvo disminución en las concentraciones de COD presentes en el agua.

Se establecieron parámetros de diseño para la planta, ya pesar de que el caudal de diseño es tan bajo es posible diseñar una planta de tratamiento de agua potable de funcionamiento hidráulico cumpliendo con las condiciones y normas establecidos por el RAS

La reducción de materia orgánica disuelta resulta mas eficiente con la aplicación de permanganato de potasio que con peróxido de hidrogeno.

\section{BIBLIOGRAFIA}

Angulo, W. J., Mendoza, J. A. y Uriel, H. $U$. (2017). Análisis de la vulnerabilidad por fenómenos de remoción en masa en la Cuenca Tanauca estudio de caso. Revista Ambiental Agua, Aire y Suelo. ISSN 19009178. Volumen (8), Numero (2). DOI: https://doi.org/10.24054/19009178.v2 .n2.2017.3276

Camargo, W. C. (2016). Modelación hidrologico-hidraulica de eventos de inundacion en el Rio Bogotá (sector tocanzipa-chia) usando HEC-RAS. Revista Ambiental Agua, Aire y Suelo. ISSN 19009178. Volumen (7), Numero (2).
DOI: https://doi.org/10.24054/19009178.v2 .n2.2016.3267

Cardeño, F., et al., Síntesis de Resinas Alquídicas a partir de Aceites de Higuerilla, de Palma y de Fritura, Mezclados con Aceite de Soja. Información tecnológica, 2013. 24(4): p. 7-8.

Espinel, R. M., Rivera, H. U. y Castellanos, C. (2016). Plan de manejo ambiental para los proyectos de extracción de material de arrastre, localizados en las microcuencas quebradas volcán y navarro del municipio de Pamplona, Norte de Santander. Revista Ambiental Agua, Aire y Suelo. ISSN 19009178. Volumen (7), Numero (1).

DOI: https://doi.org/10.24054/19009178.v1 .n1.2016.3282

Gutiérrez, T., Castellanos, C. y Hernández, N. (2016). El ordenamiento territorial frente a las consecuencias de los cambios climáticos. Revista Ambiental Agua, Aire y Suelo. ISSN 1900-9178. Volumen (7), Numero (2). DOI: https://doi.org/10.24054/19009178.v2 .n2.2016.3338

Ivanova, Y. y Sarmiento, A. (2014). Evaluación de la huella hídrica de la ciudad de Bogotá como una herramienta de la gestión del agua en el territorio urbano. Revista Ambiental Agua, Aire y Suelo. ISSN 1900-9178. Volumen (4), Numero (2), 1-5. DOI: https://doi.org/10.24054/19009178.v2 .n2.2013.427

Melo, J., Saavedra, S. y Ramón, J. (2017). Evaluación de la adsorción de cu+2 y azul de metileno en biosorbentes de bajo costo obtenidos a partir de biomasa residual de la agroindustria de cítricos. Revista Ambiental Agua, Aire y Suelo. Volumen (8), Numero (2). DOI: https://doi.org/10.24054/19009178.v2 .n2.2017.3277

Mejía, R. J (2010). Generalidades de la planta 
de potabilización del Canton Gualaquiz. Universidad de cuenca.

Meneses, V. B., Álzate, D. y Mosquera, J. (2016). Sistema de optimización de las técnicas de planificación en agricultura de precisión por medio de drones. Revista Ambiental Agua, Aire y Suelo. ISSN 19009178. Volumen (7), Numero (2). DOI: https://doi.org/10.24054/19009178.v2 .n2.2016.3268

Ministerio de Desarrollo Económico Dirección de Agua Potable y Saneamiento Básico, 2015. Reglamento Técnico Del Sector De Agua potable Y Saneamiento Básico. Titulo C: Sistemas de Potabilización. Bogotá. Colombia. Pag 2526, 29, 32, 34,52-53, 77, 100-102.

Moreno, C. y Rueda, L. (2016). La educación ambiental como herramienta para la recuperación de la cobertura vegetal, mediante prácticas agroecológicas en la comunidad minera asograstorres, asociación de gravilleros de Sabana de Torres. Revista Ambiental Agua, Aire y Suelo. ISSN 1900-9178. Volumen (7), Numero (1). DOI: https://doi.org/10.24054/19009178.v1 .n1.2016.3260

Orellana. J A. (2005). Ingeniería SanitariaUniversidad Tecnológica Nacional FRRO. Tratamiento de las aguas. Santa Fe, Argentina. Pág. 28-31, 83

Patel, V.C., et al., Synthesis of alkyd resin from jatropha and rapeseed oils and their applications in electrical insulation. Journal of Applied Polymer Science, 2008. p. 1724-1729.

Pérez P, J A. (2009). Manual de potabilización del agua. Medellín: Universidad Nacional de Colombia. Colombia.

Rodríguez, Y. A. (2017). Conservación de humedales en el marco de gestión de cuencas hidrográficas. Puerto RondónArauca. Revista Ambiental Agua, Aire y Suelo. ISSN 1900-9178. Volumen (8), Numero DOI: https://doi.org/10.24054/19009178.v2 .n2.2017.3281

Rojas, V. J (2008). Potabilización de aguas superficiales mediante el proceso de ultrafiltración con membranas arrolladas en espiral. Universidad De Granada. Departamento de Ingeniería Civil.

Pouchert, C.J., The Aldrich Library of Infrarred Spectra, (1981). 3rd ed.. Aldrich Chemical Co, Milwakee, 1873

ROMERO, R. J A. 2000. Purificación del agua. Bogotá: Escuela Colombiana De Ingeniería Colombia.

Vanegas, D., Ramón, A. A. y Lizarazo, A. K. (2017). Comunidad y cultura ambiental. dinámicas de potenciación para un desarrollo sostenible y corresponsable. Revista Ambiental Agua, Aire y Suelo. ISSN 1900-9178. Volumen (8), Numero (1).

DOI: https://doi.org/10.24054/19009178.v1 .n1.2017.3271

Velandia, F. J., Granados, J. D., Ramón, J. D. y Roa, A. L. (2016). Caracterización de consorcios microbianos con potencial degradador de contaminantes en el municipio de Pamplona, Norte de Santander. Revista Ambiental Agua, Aire y Suelo. ISSN 1900-9178. Volumen (7), Numero DOI: https://doi.org/10.24054/19009178.v1 .n1.2016.3278

Villamizar, V. A. y Justinico, A. J. (2017). Reconstrucción paleoclimatica y paleoambiental de los territorios de la llanura inundable del araucana a partir del análisis de sedimentos recientes, Departamento de Arauca. Revista Ambiental Agua, Aire y Suelo. ISSN 19009178. Volumen (8), Numero (1). 
DOI: https://doi.org/10.24054/19009178.v1 .n1.2017.3272

Zagorodni A.A., KOTOVA L.D. Y
SELEMNEV F.V.Infrared spectroscopy of ion Exchange resins: chemical deterioration of the resins. React. Funct. . 2002. Pol. 53, 157-171. 